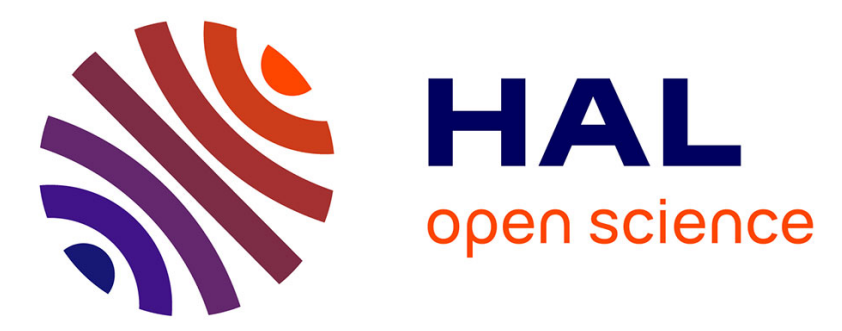

\title{
Eva e Avrai i miei occhi: tracciare e ricomporre le mappe sulla pelle.
}

Monica Jansen, Claudio Milanesi

\section{To cite this version:}

Monica Jansen, Claudio Milanesi. Eva e Avrai i miei occhi: tracciare e ricomporre le mappe sulla pelle.. Narrativa, 2021, La fantascienza nelle narrazioni italiane contemporanee, 43, pp.235-257. 10.4000/narrativa.413 . hal-03501480

\section{HAL Id: hal-03501480 \\ https://hal-amu.archives-ouvertes.fr/hal-03501480}

Submitted on 23 Dec 2021

HAL is a multi-disciplinary open access archive for the deposit and dissemination of scientific research documents, whether they are published or not. The documents may come from teaching and research institutions in France or abroad, or from public or private research centers.
L'archive ouverte pluridisciplinaire HAL, est destinée au dépôt et à la diffusion de documents scientifiques de niveau recherche, publiés ou non, émanant des établissements d'enseignement et de recherche français ou étrangers, des laboratoires publics ou privés. 


\section{narrativa}

nuova serie, n. $43-2021$

\section{La fantascienza nelle narrazioni italiane ipercontemporanee}




\title{
La fantascienza nelle narrazioni italiane ipercontemporanee
}

\author{
A cura di
}

Daniele Comberiati e Luca Somigli 
Italies

NARRATIVA

rivista fondata da Marie-Hélène CASPAR

NARRATIVA nuova serie

Direttrice : Silvia CONTARINI

Vice-direttrice : Giuliana PIAS

Segretario di redazione : Alessandro BeNUCCI

\section{Comitato scientifico e di lettura}

Giuliana Benvenuti, Bologna (Italia)

Vincenzo BinetTi, Ann Arbor, Michigan (USA)

Alberto CASAdei, Pisa (Italia)

Paolo Chirumbolo, Baton Rouge (USA)

Laura Di Nicola, Roma (Italia)

Monica JANSEN, Utrecht (Paesi Bassi)

Peter Kuon, Salisburgo (Austria)

Stefano LAZZARIN, Saint-Étienne (Francia)

Federico Luisetti, St. Gallen (Svizzera)

Margherita MArras, Avignone (Francia)

Donata Meneghelli, Bologna (Italia)

Claudio Milanesi, Aix-en-Provence (Francia)

Giuseppe Nicoletti, Firenze (Italia)

Ramona OnNIS, Nanterre (Francia)

Hanna Serkowska, Varsavia (Polonia)

Patrizia SERRA, Cagliari (Italia)

Beatrice SicA, Londra (UK)

Luca Somigli, Toronto (Canada)

Bart Van Den Bossche, Lovanio (Belgio)

(C) Presses universitaires de Paris Nanterre issn: $1166-32-43$

isbn: 978-2-84016-485-2 


\section{Sommario}

La fantascienza nelle narrazioni italiane ipercontemporanee

Daniele Comberiati e Luca Somigli

La fantascienza italiana dalla prospettiva degli studi sulla traduzione e postcoloniali

Simone BRIONI

Sognando la catastrofe. L'eco-distopia italiana del ventunesimo secolo

Marco Malvestio

Fantascienza femminista

Giuseppe CARrARA

Dalla Madre alle madri? Fra distopia, mito e realtà

Ramona OnNis e Manuela SpINELLI

Fantascienza italiana young adults

Beatrice LAGHEZZA

I bambini dell'apocalisse. Racconti della fine e di nuovi inizi nella fantascienza italiana degli anni Duemila.

Simona Micali

Dei bambini non si sa niente? L'infanzia come altrove distopico in Anna (Ammaniti), Bambini Bonsai (Zanotti), La terra dei figli (Gipi) e L'uomo verticale (Davide Longo)

Valentina FuLginiti

Hantologia e fantarcheologia in Medium di Giuseppe Genna.

Fabio CAmilLetTi 
L'originale di Paolo. La fantascienza nell'opera narrativa di Paolo Zanotti. 141

Stefano LAZZARIN

Post-umano, troppo post-umano: Sirene di Laura Pugno

Pierpaolo ANTONELLO

Speculative-evolution fiction, quantum-actualist fiction:

letture di Laura Pugno

Hanna Serkowska e Aleksandra PŁawska

Ibridità testuale e ibridità creaturale in Sirene di Laura Pugno

Amélie AuberT-NoËL

Contro-passato prossimo: genesi di una storia alternativa

in La nostra guerra di Enrico Brizzi.

Umberto Rossi

Speculazioni ecologiche:

impegno e retrotopia nel romanzo italiano contemporaneo

Florian Mussgnug

"Un'orrenda vita da vivere": la leggenda privata di

Michele Mari fra autofiction e SF.

Stefania LUCAMANTE

Eva e Avrai i miei occhi: tracciare e ricomporre le mappe sulla pelle

Conversation pieces

Monica Jansen e Claudio MilanesI

Lo chiamavano Jeeg Robot: un supereroe italiano tra eroismo e decadenza.

Anna Chiara PALLAdino

Lupo siderale: il connettivismo di Giovanni De Matteo

e la fantascienza italiana. Con un'intervista a Giovanni De Matteo

Arielle SAIBER

Recensioni. 
Questo numero della rivista si propone di mappare le produzioni fantascientifiche italiane iper-contemporanee, un contesto variegato, molteplice e estremamente vivace. La nostra riflessione nasce dalla definizione della fantascienza italiana come un genere "aperto", all'interno del quale coabitano sottogeneri quali le narrazioni distopiche e eco-distopiche, l'ucronia, i testi post-catastrofisti, e capace di rappresentare le problematiche più attuali della società. Gli articoli inclusi nel volume mostrano inoltre delle precise tendenze e dei particolari approcci teorici: il postumanesimo e l'ecocritica, come l'attenzione alle teorie femministe, alla transmedialità e alle relazioni con altri contesti culturali, nonché la capacità di costruire e decostruire costantemente il proprio canone di riferimento.

\section{RÉSUMÉ DU VOLUME}

Ce numéro de la revue a pour objectif de cartographier les productions italiennes de science-fiction hyper-contemporaines, un contexte varié, multiple et extrêmement vivant. Notre réflexion part de la définition de la science-fiction italienne comme un genre "ouvert", capable aussi de représenter les enjeux de société les plus actuels, au sein duquel coexistent des sous-genres tels que les récits dystopiques et éco-dystopiques, l'uchronie, les textes post-catastrophiques. Les articles réunis dans ce volume montrent également des tendances précises et des approches théoriques particulières : le posthumanisme et l'écocritique, l'attention aux théories féministes, à la transmédialité et aux relations avec d'autres contextes culturels, ainsi que la capacité à construire et déconstruire constamment les modèles de référence. 


\title{
Eva $e$ Avrai i miei occhi: tracciare e ricomporre le mappe sulla pelle Conversation pieces
}

\begin{abstract}
RiASSUNTO
Ispirati dalla complementarietà tra Nigredo e Olivia, due personaggi a metà che hanno bisogno degli occhi dell'uno e dell'empatia dell'altra per ricomporre i mosaici di cui sono fatti, abbiamo ideato questo contributo su Avrai $i$ miei occhi di Nicoletta Vallorani come una serie di conversation pieces, non a caso il titolo di uno dei capitoli del romanzo. Un dialogo non solo tra di noi, e tra le nostre competenze su noir, (micro)storia, postmoderno e transmedialità, ma anche una conversazione tra i romanzi di Vallorani che ambiscono a formare una trilogia in progress: Eva (2002), ripubblicato di recente in una nuova edizione allargata, e Avrai i miei occhi (2020).
\end{abstract}

\section{RÉSUMÉ}

Inspirés par la complémentarité entre Nigredo et Olivia, deux personnages à moitié qui ont besoin des yeux de l'un et de l'empathie de l'autre pour recomposer les mosaïques dont ils sont faits, nous avons conçu cette contribution sur Avrai $i$ miei occhi de Nicoletta Vallorani comme une série de conversation pieces, sans surprise le titre de l'un des chapitres du roman. Un dialogue non seulement entre nous, et entre nos compétences sur roman policier, (micro)histoire, postmodernisme et transmédialité, mais aussi une conversation entre les romans de Nicoletta Vallorani qui aspirent à former une trilogie in progress: Eva (2002), récemment republié dans une nouvelle édition agrandie, et Avrai i miei occhi (2020).

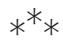

Ispirati dalla complementarietà tra Nigredo e Olivia, due personaggi a metà che hanno bisogno degli occhi dell'uno e dell'empatia dell'altra per ricomporre i mosaici di cui sono fatti, abbiamo ideato questo contributo su Avrai $i$ miei occhi come una serie di conversation pieces, non a caso il titolo di uno dei capitoli del romanzo. Un dialogo non solo tra di noi, e tra le nostre competenze su noir, 
(micro)storia, postmoderno e transmedialità, ma anche una conversazione tra i romanzi di Nicoletta Vallorani che ambiscono a formare una trilogia in progress: Eva (2002), ripubblicato di recente in una nuova edizione allargata, e Avrai i miei occhi $(2020)^{1}$ condividono personaggi, città e trame e una cronologia consecutiva che porta dall'anno 2023 di Eva agli anni 2029-2030 di Avrai i miei occhi. Ambedue quindi narrazioni ambientate in un futuro che progressivamente si evolve verso un antropocene sempre più postumano (Eva), mentre il passato sopravvive nelle finte rievocazioni del centro storico di Milano con l'aiuto di ologrammi (Avrai i miei occhi). L'epigrafe dell'edizione del 2002 di Eva destina il libro a "chi ha perso la memoria, per volontà o per caso". Avrai i miei occhi è invece concepito come un doppio omaggio, "Per Beppe Devalle, il pittore cui devo la bellezza dell'arte e la magia dell'ispirazione", e "per le donne. Per le ragazze che conservano i loro sogni e li agiscono. Per le invisibili che portano sulle spalle il mondo, e che il mondo, spesse volte, dimentica". Sempre di memoria si tratta, ma questa volta $i$ lettori vengono invitati a tradurre la perdita in una pratica estetica e sociale, di immaginare nuovi modi di stare nel mondo.

1. La prima domanda che possiamo porci è di quale tipo di fantascienza si tratti in questi libri che si iscrivono esplicitamente nel genere ma le cui visioni sembrano costruirsi nell'assenza di mondi possibili e proporre poco di futuribile. Vallorani ripete in vari interventi che la letteratura di genere le permette di combinare intrattenimento e speculazione, e di parlare di aspetti scomodi dell'attualità rendendoli visibili attraverso la lente dello straniamento. Nei "Ringraziamenti" che chiudono Avrai i miei occhi Vallorani definisce la sua "piccola storia" come "fantascienza" e afferma di condividere la sua "scelta di "fare fantascienza" con un gruppo di amiche scrittrici sulla scia di Daniela Piegai", "stella polare e punto di riferimento in questo campo". In che modo le narrazioni fs di Eva e Avrai i miei occhi corrispondono a queste interpretazioni e usi del genere?

\section{Claudio MilanesI}

Per chi non ha mai letto i suoi romanzi, è forse utile richiamare subito certi classici cui viene fatto di pensare leggendola. È una fantascienza che scrive del domani per parlare dell'oggi, per svelare attraverso un’idea di futuro tendenze

1. Vallorani Nicoletta, Eva. Nuova edizione [2002], Modena, Zona 42, 2021; EAD., Avrai i miei occhi, Modena, Zona 42, 2020.

2. Simone Brioni e Daniele Comberiati (Ideologia e rappresentazione. Percorsi attraverso la fantascienz̧a italiana, Mimesis, Milano, 2020, p. 22) indicano Il mondo non è nostro di Daniela Piegai (1989) come esempio della fantascienza italiana degli anni Ottanta che "descrive un mondo che ha minore speranza in un miglioramento futuro". 
già iscritte nelle dinamiche del presente. Straniamento, speculazione, certo, ma anche critica sociale e politica dell'esistente. È per questo che vengono in mente Metropolis (1926) di Thea Von Harbou tradotto in film dal marito Fritz Lang (1927), 1984 di George Orwell (1947), adattato al cinema da Michael Radford proprio nel 1984, Blade Runner (1982) di Ridley Scott ispirato dal Cacciatore di androidi (1968) di Philip K. Dick e La strada (2006) di Cormac Mc Carthy diventato poi il film di John Hillcoat (2009). Insisto sul doppio riferimento letterario e filmico non solo per ossessione documentaria ma perché nell'immaginario di Vallorani si colgono riferimenti sia ai romanzi che alle loro riletture cinematografiche. E ci insisto anche per ricordare che se accettiamo l'ipotesi che la fantascienza l'abbia inventata una scrittrice, Mary Shelley, con Frankenstein o il Prometeo moderno (1818 e 1831), il nome di Thea Von Harbou, attrice, scrittrice e sceneggiatrice, in questa genealogia femminile del genere è troppo spesso dimenticato, senza dubbio per la sua conversione al nazismo nel 1932.

Parlerei di distopia, innanzitutto. Un mondo futuro frutto dell'utopia malata e del progresso distorto. Ci sono esempi molto concreti in Avrai i miei occhi, i cui personaggi centrali sono cavie e cloni frutto di sperimentazioni genetiche: inizialmente, si racconta, gli esperimenti erano stati finalizzati al miglioramento delle condizioni di vita della specie (immunità, resistenza ai processi di invecchiamento), poi hanno avuto la meglio le tendenze perverse del mercato e dell'interesse mescolate al soddisfacimento del piacere fondato sulla sopraffazione. Le finalità etiche della scienza si sono pervertite. La ricerca del profitto e la sopraffazione hanno prevalso sul miglioramento delle condizioni individuali e collettive.

In un altro momento, la voce narrante, in questo caso la taxista Olivia, riflette sulla perversione degli idealismi egualitari degli anni della sua giovinezza, e pensa: "i cloni hanno rappresentato la perdita di singolarità, il miraggio di un mondo di uguali: quello che ci aveva abbacinato giusto prima dello Split" ${ }^{3}$, un termine con cui viene definito il colpo di Stato che ha portato alla suddivisione della città in quartieri ermeticamente (o quasi) chiusi. Anche qui, gli ideali, in questo caso quelli di giustizia e di eguaglianza, finiscono con il pervertirsi, nella Milano distopica dei primi anni '30 del XXI secolo, e diventano pratica di clonazione di corpi (quelli sì letteralmente tutti uguali) votati al soddisfacimento del piacere di chi se li può procurare e può disporne liberamente. Ma è chiaro che la metafora della perversione degli ideali, scrivendo del domani, parla dell'oggi: traffico di organi, abusi della chirurgia estetica, dominio del mercato

3. Vallorani Nicoletta, Avrai i miei occhi, cit., p. 134. 
delle società neoliberali, violenza sulle donne, oggettivazione e reificazione dei loro corpi. Come il mondo di Orwell, che sembrava parlasse del 1984, ma stava descrivendo in modo condensato i totalitarismi del Novecento.

\section{Monica JANSEN}

Vallorani viene infatti considerata come esponente del sottogenere del cyberpunk che caratterizza la fantascienza in Italia dei primi anni Ottanta e presenterebbe una visione radicale e politica di un futuro tecnologico e in decadenza ${ }^{4}$. Il genere sarebbe anche stato usato per esprimere una critica sul fallimento politico dei movimenti sociali alla fine degli anni Settanta ${ }^{5}$ attraverso uno dei suoi organi, la rivista del collettivo Un'ambigua utopia ${ }^{6}$ fondata nel 1977, che mette in relazione anarchia e fantascienza. Tra i temi del cyberpunk, di cui uno dei primi esempi è Neuromancer (1984) di William Gibson ${ }^{7}$, vi è quello del cyborg, che è composto da parti umane e artificiali e quindi rappresenta una sfida terrificante per la distinzione tra "copia" e "originale", una tematica ricorrente nelle finzioni di Vallorani. Inoltre, l'ossessione per le mutazioni genetiche spesso sta in stretta relazione con la predominanza di personaggi marginali e alienati, appartenenti a quelle classi sociali che causano un radicale cambiamento nell'ordine sociale?. Anche quest'aspetto è presente sia in Eva, dove l'assassina Eva opera insieme a un gruppo di marginali da lei gestiti come direttrice del Centro di Riabilitazione ("cr come Centro Rifiuti"10), sia in Avrai i miei occhi, dove il mistero del massacro delle cavie viene risolto da personaggi collocati fuori dall'ordine imposto dal Profeta. Vallorani dice in un'intervista che l'ambientazione

4. Brioni Simone, Comberiati Daniele, Italian Science Fiction. The Other in Literature and Film, Cham, Palgrave Macmillan, 2019, p. 168.

5. Ibid.

6. Il titolo riprende un romanzo di Ursula Le Guin, di cui Vallorani scrive un necrologio nel 2018: "La grandezza di Ursula K. Le Guin, icona immortale del fantasy e della fantascienza", Nero, 29 gennaio 2018, https://not.neroeditions.com/ursula-le-guin-nicoletta-vallorani/. Su l'A rivista anarchica Vallorani ha una rubrica, intitolata La guida Apache. La rivista ha pubblicato nel 1998 un numero speciale per ricordare il collettivo Un'Ambigua Utopia, a cui Vallorani ha contributo con il racconto "Libero in freezer. Ricordando Piazza Fontana", ripubblicato nella raccolta Si muore bambini, Perdisa, Città di Castello, 2008, pp. 106-110.

7. Vallorani ha contribuito a diffondere l'opera di Gibson in Italia con le sue traduzioni; si veda il catalogo https://www.fantascienza.com/catalogo/autori/ NILF12216/william-gibson/.

8. Brioni Simone, Comberiati Daniele, Italian Science Fiction, cit., p. 165.

9. Ibid., p. 167.

10. Vallorani Nicoletta, Avrai i miei occhi, cit., p. 62. 
postindustriale è da considerare un "omaggio all'iconografia urbana del cyberpunk"11.

Vallorani è stata la prima donna a vincere il Premio Urania nel 1992 con Il cuore finto di $\mathrm{DR}$, e ha continuato a scrivere letteratura di genere alternamente classificata come noir, SF, o fantasy e associata, come suggerisce Silvia Contarini, ai "romanzi [...] femministi, al cui centro stanno tormentate storie di donne, oggetto di rapporti di oppressione, costrette in ruoli sociali imposti con la forza dell'ordine simbolico e fisico"12. A queste proporrei di aggiungere altre due categorie adatte per i romanzi di cui qui discutiamo. Una potrebbe essere quella di black novel, un concetto ideato dal fondatore del noir inglese Derek Raymond nel testo autobiografico The Hidden Files (1992), che Vallorani riassume come una definizione allargata del noir in cui viene enfatizzata la nozione di frizione sociale o sociologica, e che mette in scena il gioco finale tra bene e male sul crocevia oscuro tra vita e morte. L'associazione di questa definizione ampliata del genere a romanzi che trattano dell'abuso sessuale del corpo femminile la rende particolarmente adatta per definire trama e stile di Avrai i miei occhi ${ }^{13}$. L'altra categoria è quella di fairy-tale noir, concetto coniato da Danila Cannamela per rubricare le fiabe dark di una serie di scrittrici associate al noir italiano. Il trauma incarnato dalle categorie vulnerabili rappresentate in queste storie generebbe catene infinite di violenza, visualizzate da topoi e immagini di derivazione dark fantasy e noir, narrate con una prospettiva che ora sta dalla parte delle vittime, e ora abbraccia il "punto di vista di Caino"14.

"Caino, cos'hai fatto a tuo fratello? Se Abele fosse nato oggi sarebbe una donna. E di sicuro una di queste ragazze" 15 : per Vallorani la fantascienza è sempre anche romanzo femminista. In un volume recente sulle scritture tra fantastico e fantascienza, Vallorani e Anna Pasolini ipotizzano che i due generi abbiano in comune due battaglie politiche intrecciate, "una concerne la revisione, di

11. Contarini Silvia, “Avrai i miei occhi’, di Nicoletta Vallorani”, Nazione Indiana, 22 marzo 2020, https://www.nazioneindiana.com/2020/03/22/ avrai-i-miei-occhi-di-nicoletta-vallorani/.

12. Ibid.

13. Vallorani Nicoletta, 'Speaking Bodies. The 'Black Novel' and Women as Borderland Identities", in Bulfoni Clara, Lupano Emma e MotTura Bettina (a cura di), Sguardi sull'Asia e altri scritti in onore di Alessandra Cristina Lavagnino, Milano, Lingue Culture Mediazioni, 2017, pp. 402-403.

14. Cannamela Danila, "A Fairy-Tale Noir: Rewriting Fairy Tales into Feminist Narratives of Exposure", in Quaderni d'italianistica, vol. 39, n. 2, 2018, p. 88. Le scrittrici analizzate sono Laura Pugno, Nicoletta Vallorani, Simona Vinci, Alda Teodorani.

15. Vallorani Nicoletta, Avrai i miei occhi, cit., p. 218. Corsivo nel testo. 
necessità profonda, del rapporto tra maschile e femminile; l'altra allarga l'orizzonte, proponendo una ricomposizione - forse tardiva e senz'altro necessaria - del rapporto tra gli esseri umani e lo scenario che essi abitano" ${ }^{16}$.

In un'intervista Vallorani osserva, a proposito dell'evoluzione del genere nel presente:

È interessante come scrittrici e scrittori di fs tra i più interessanti di oggi continuino a tirar fuori ipotesi legate alla creazione di una rete tra organismi e natura, riprendano in mano la questione del rispetto, immaginino una libertà di matrice anarco-pacifista: si può essere liberi nel mutuo rispetto della libertà degli altri. È una componente che trovi in Nora K. Jemisin come in Sam J. Miller ${ }^{17}$.

È questo sviluppo verso la finzione speculativa che ha portato Vallorani a pubblicare con la casa editrice di fantascienza Zona $42^{18}$, che punta su lettori pronti a una SF caratterizzabile, secondo Evangelisti, come "difficile" o "letteraria""

2. Avrai i miei occhi può essere letto come suite di Eva, e ciò spiega perché Zona 42 ne pubblichi una nuova edizione ampliata, in cui, oltre alla prefazione della "Booktuber" Tiffany Veccbietti, a una nota dell'autrice, ai tre racconti, "SnuffMovie", "Shangri-La", "Taboulhe", si nota l'inserimento nell'originale di Eva di monologhi in corsivo di personaggi che nella versione precedente non avevano voce ${ }^{20}$. Possiamo chiederci come interagiscono i paratesti e gli intratesti nelle tessiture delle trame di Vallorani.

16. Pasolini Anna, Vallorani Nicoletta, Corpi magici: Scritture incarnate dal fantastico alla fantascienza, Milano, Mimesis, 2020, p. 11.

17. Braggion Romina, “Avrai i miei occhi’ romanzo di Nicoletta Vallorani”, diario di errebi, 23 gennaio 2020, https://rominabraggion.blogspot.com/2020/01/avrai-imiei-occhi-romanzo-di-nicoletta.html.

18. Zona 42 pubblica anche Alessandro Vietti, esponente del cyberpunk italiano degli anni Ottanta. Sul sito di Zona 42, si legge: "riteniamo la fantascienza uno degli strumenti più utili a riflettere sulla contemporaneità, senza per questo rinunciare alla capacità d'intrattenimento insita al genere", http://www.zona42.it/wordpress/ chi-siamo/

19. Evangelisti Valerio, "Nell'inverno di Milano spuntano cadaveri. È un cimitero di donne o replicanti?”, La Stampa Tuttolibri, 29 febbraio 2020, disponibile su http:/ / www.zona42.it/wordpress/avrai-i-miei-occhi-recensioni-3-tuttolibri/

20. Si noti che Vallorani non tiene a menzionare la pubblicazione originale dei suoi testi che lascia viaggiare da un'antologia a un blog a un romanzo, all'interno di una macronarrazione senza confini di storie e di genere. Di "Snuffmovie" menzioniamo almeno due pubblicazioni precedenti, nell'antologia Tutti $i$ denti del mostro sono perfetti, a cura di Valerio Evangelisti (Mondadori, Milano, 1997), e nell'antologia di Vallorani Si muore bambini (cit.). "Taboulhe" fa parte dei tre racconti ambientati nella Milano del futuro raccolti da Vallorani in Il catalogo delle vergini (Future Fiction, 2017). "Shangri-La" 
Claudio Milanesi

È l'aspetto che più ha provocato il mio "straniamento" quando invece di leggerli ho cominciato a studiarne la composizione e le connessioni. Forse è una mia deformazione, venuta da altre mie letture, ma, in sintesi, ho l'impressione che quella di Vallorani sia una sola grande opera globale in cui temi, forme, personaggi e ossessioni vanno e vengono, si rimandano gli uni agli altri, appaiono e scompaiono da una narrazione all'altra. È quello che tu chiami l'“intratesto", in cui i rimandi interni da un testo all'altro servono a saldare i vari romanzi in un grande disegno, non al modo di Guerre stellari cioè di pezzi di cronologia che si incastrano successivamente, ma più nel senso in cui componeva, per fare un esempio, lo scrittore cileno Roberto Bolaño, nella cui opera labirintica temi e personaggi di un romanzo riappaiono inaspettatamente in un altro, in altri contesti e altre temporalità. Nel mondo narrativo di Vallorani, i richiami di questo tipo sono molteplici; ne faccio due esempi: ritroviamo un determinato modo operativo, che consiste nel comporre i cadaveri delle vittime come opere d'arte, sia nel suo primo noir Dentro la notte e ciao ${ }^{21}$ che in Eva; la presenza di personaggi ibridi e meticci con nomi di fantasia appare già in Cuore meticcio $^{22}$, un noir della serie di Zoe Libra, per ricomparire poi in Avrai i miei occhi, dove appaiono un Nikon, un Nigredo (già voce narrante e protagonista di Eva), una Kio e una Kariel.

Monica Jansen

Con l'esempio della figura di Eva è possibile far vedere come paratesti e intratesti s'intrecciano nella scrittura di Vallorani. Nella nuova edizione di Eva leggiamo in epigrafe della prefazione: "Di qua dal muro, Eva non ha mai mangiato la mela e non è mai stata scacciata dall'Eden”. Tiffany Vecchietti cita da Avrai $i$ miei occhi e commenta in un post: "Stavo leggendo Avrai $i$ miei occhi di Nicoletta Vallorani e sei ricomparsa tu. Ho pensato alla tua lezione, a ciò che ci hai lasciato. Avrei sperato in una realtà meno sconfortante e più trasformata, avrei voluto vedere dei cambiamenti più dirompenti, li aspettavamo"23.

Per mettere questa frase in prospettiva, partirei dal volume Mappe sulla pelle del collettivo Tessere Trame, concepito nel 2012 e ripubblicato sul blog di Sdiario

è anche in rete sul blog Sdiario di Barbara Garlaschelli, 27 gennaio 2020, https:/ / sdiario.com/shangri-la-di-nicoletta-vallorani/

21. Vallorani Nicoletta, Dentro la notte e ciao, Bologna, Granata Press, 1995.

22. EaD., Cuore meticcio, Milano, Marcos y Marcos, 1998.

23. Vecchietti Tiffany, "File_Eva01", in Vallorani Nicoletta, Eva, cit., p. v. La citazione da Avrai $i$ miei occhi è a p. 34. 
nel $2019^{24}$. La raccolta si apre con il testo programmatico "Una voce tutta per sé" che si sofferma tra l'altro sulla "costola di Adamo":

C'è un dato che non bisogna dimenticare: la storia delle donne si lega al loro corpo, fatalmente. C'è una fisicità invadente che definisce l'esperienza femminile e la connota, nella cultura occidentale, come 'derivata'. Siamo nate dalla costola di Adamo, dicono. Siamo una creazione di secondo grado, fatta di avanzi e funzionale al maschio della specie. Siamo destituite di autonomia, schiave della luna, condannate e benedette dalla maternità, deboli, violabili, emotive. Siamo, in altri termini, fisicamente inabili all'autonomia ${ }^{25}$.

Il volume contiene dieci storie di scrittrici a cui è stato chiesto di mettersi nei panni del loro profilo femminile modello. Vallorani, con la storia "Camere di sangue", si è modellata su Angela Carter e scrive con la sua voce:

E se fosse possibile capire cosa significa avere un corpo di donna? Se un uomo si trovasse, a un certo punto della vita con un corpo di donna? La mia nuova Eva era un uomo prima che Mother gli donasse un corpo di donna. Come una punizione, una condanna per espiare il peccato di aver abusato del suo potere. Sperimentare lo stupro, la violenza, la maternità, la nascita. La colpa primordiale di essere donna, e, dunque, violabile. Il corpo è la chiave ${ }^{26}$.

Il riferimento è al romanzo The Passion of the New Eve (1982) a cui Vallorani ha dedicato un articolo nel 199427. Nel romanzo di Carter, la cui visione artistica viene associata da Vallorani al "female gothic" 28 , il corpo del protagonista maschile Evelyn viene trasformato in quello di una donna, per cui diventa Eve. Nasce così un ibrido tra uomo e donna che risulta in due entità contigue che però non possono integrarsi in una nuova continuità. Il risultato è una disposizione caotica con un corpo e una mente divergenti che insieme provocano un processo di comprensione (e non una fusione) tra $\mathrm{i}$ due sessi ${ }^{29}$. Eve/lyn

24. Tessere Trame (a cura di), Mappe sulla pelle, Firenze, Editpress, 2012; "Mappe sulla pelle. Un ritorno", Sdiario, 11 maggio 2019, https://sdiario.com/ mappe-sulla-pelle-un-ritorno/

25. Ibid.

26. Vallorani Nicoletta, "Camere di sangue", Sdiario, 18 maggio 2019, https:// sdiario.com/camere-di-sangue-di-nicoletta-vallorani/

27. Vallorani Nicoletta, "The Body of the City: Angela Carter's 'The Passion of the New Eve"”, in Science Fiction Studies, n. 21, 3, 1994, pp. 365-379.

28. Ibid., p. 369.

29. Ibid., p. 368. 
acquisterà coscienza della sua metamorfosi grazie al dolore e all'umiliazione provocati dalla violenza e dall'abuso sessuale che sentirà perpetrati sul proprio corpo $^{30}$. Si tratta in altre parole di una sexed utopia, un genere con un gender femminile ${ }^{31}$.

Tutto questo fa vedere come la "personaggia"32 di Eva stia in diretta corrispondenza con le sue valenze metacritiche senza per questo perdere la sua autonomia finzionale. In Eva la donna vestita di nero quando è assassina e di bianco quando è salvatrice, tiene ben distinta la sua doppia personalità, mentre in Avrai $i$ miei occhi, nel ricordo di chi l'ha conosciuta e amata, diventa l'ossimorica "benefattrice assassina" ${ }^{33}$. Se in Eva era Eva l'altra metà della rabbia di Nigredo $^{34}$, in Avrai i miei occhi è Olivia che, con la sua empatia, darà a Nigredo la visione per comprendere il dolore e porci fine con un atto estremo. L'azione vendicatrice di Eva in Avrai i miei occhi verrà portata avanti da Ariel, altra personaggia che, con un nome shakespeariano, travalica i confini del macrotesto valloriano. Il legame più diretto è con il racconto "SnuffMovie", ma c'è una Ariel anche nel romanzo Le madri cattive in cui indossa i panni di una psichiatra che ripara "anime segnate dalla nevrosi, dalla depressione, dalla follia. Soprattutto di donne" ${ }^{\text {". Si }}$ Si tratta dunque per Vallorani di tracciare con la finzione una "mappa sulla pelle", che salda il corpo femminile con la città, e questo ci riporta alla Eve di Carter.

Vallorani spiega come nel romanzo di Carter vengano attraversate diverse città/comunità utopiche/distopiche che si dispongono come labirinti, reti di simboli privi di significato, e la cui preferenza cromatica per il buio sia funzionale per la rappresentazione di un caleidoscopo urbano spoglio di qualsiasi

30. Ibid., p. 367.

31. Ibid., p. 365.

32. Pasolini e Vallorani specificano di utilizzare il neologismo "personaggia" introdotto dalla Società Italiana delle Letterate (SIL) perché "sancisce un nuovo punto di vista sul femminile nella letteratura e sulla letteratura stessa, non più (e non solo) come 'corrispondente' o 'complementare' al maschile"; Pasolini Anna, Vallorani Nicoletta, Corpi magici, cit., p. 11.

33. Vallorani Nicoletta, Avrai i miei occhi, cit., p. 106.

34. Si veda il capitolo "Due voci", un duetto in corsivo tra Eva e Nigredo: "Voglio le tue mani. Voglio i tuoi occhi. Voglio il tuo cuore. Voglio te, per intero. Per curare la mia solitudine. / Voglio il tuo rancore, la tua rabbia, il tuo dolore, quello che avresti potuto essere e quello che sei. Per curare la mia solitudine" (VAllorani Nicoletta, Eva, cit., p. 267).

35. Braggion Romina, "Recensione: Le madri cattive", diario di errebi, 2 aprile 2020, https:/ / rominabraggion.blogspot.com/2020/04/recensione-recensione-le-madri-cattive.html. 
piano o disegno razionale ${ }^{36}$. Vallorani ipotizza un'analogia e una sorta di contiguità tra il corpo fisico di una persona e il corpo urbano di una città, di modo che si possano leggere i segni nello spazio urbano come le cicatrici sulla pelle ${ }^{37}$. Questo paesaggio urbano, all'interno delle sue delimitazioni, è configurato come un corpo diluito che alla fine è un corpo sessuato ${ }^{38}$ che mette in questione la relazione tra soggettività femminile e sistema patriarcale.

Le descrizioni delle città future visionate da Carter riportano direttamente all'immaginario di Vallorani, perfino ai nomi dei suoi personaggi, come si legge in questa frase da The Passion of the New Eve citata da Vallorani, in cui il protagonista vede la città di New York come uno spazio di liminalità con una sequenza infinita di periferie in decadenza: "it was then an alchemical city. It was chaos, dissolution, nigredo, night" ${ }^{\prime 3}$. Se la Milano del Profeta immaginata in Avrai i miei occhi è da considerare una comunità patriarcale e autocratica alla stregua della città di Zero nel romanzo di Carter, allora si potrebbe supporre che Vallorani, sulla scia di Carter, si appropri del suo paradigma per decostruirlo a fini femministi $^{40}$. Vallorani afferma che la femminilità, quando è esclusa dalla storia umana, torna in superficie nell'utopia e cannibalizza lo spazio urbano, assorbendolo in un corpo femminile ${ }^{41}$. Possiamo vedere la messa in atto di quest'idea nella "sorellanza" (ri)vendicatrice che si stabilisce tra Ariel e Olivia, che infonde la loro solidarietà tra vittime di violenza e abusi sessuali anche nell'altra metà, costituita da Nigredo, di modo che nasca una comprensione tra i generi nonostante Nigredo avverta la differenza maschile a cui neanche lui può sottrarsi ${ }^{42}$.

3. Avrai i miei occhi nasce quindi anche in dialogo con il progetto collettivo di Mappe sulla pelle. Vallorani si è messa nei panni di Angela Carter, le cui tracce narrative si trovano un po' ovunque nelle sue opere. Questa è solo un'indicazione della fitta rete di rimandi intertestuali con cui Vallorani compone le sue finzioni che abbondano di riferimenti highbrow e lowbrow. L'esempio di Carter fa anche vedere il lavoro di riscrittura in chiave

36. Vallorani Nicoletta, “The Body of the City", cit., p. 370.

37. Ibid., p. 366.

38. Ibid., p. 377.

39. Ibid., p. 370, corsivo nostro.

40. Ibid., p. 375.

41. Ibid., p. 366.

42. "Tu rimani fermo, stordito non tanto dal dolore alle mani malamente tagliate, quanto da quello che hai scoperto di te, e che non avresti mai sospettato di trovarti dentro: un comportamento usuale, da maschio, di cui avresti negato anche la possibilità se qualcuno te l'avesse anticipata" (Vallorani Nicoletta, Avrai $i$ miei occhi, cit., p. 129). 
femminista delle fiabe, dei miti e della Bibbia. Come si può riconoscere questo scrivere per citazioni che somiglia all'attività artistica del tagliare a pezzi e di ricomposiz̨ione di frammenti, al centro delle storie raccontate in Eva $e$ in Avrai i miei occhi?

\section{Claudio Milanesi}

- Segmento e ricompongo: è il gioco che faccio, amico mio.

E noi? A che gioco stiamo giocando, noi? Noi, che artisti non siamo?

- Il puzzle crea una bellezza stridente, che è il nuovo. L'essenza non ancora perlustrata, che è arte ${ }^{43}$.

La poetica del Pittore è chiaramente esposta nel capitolo 15 di Avrai i miei occhi, in cui Nigredo va a far visita all'artista nel suo atelier perso nelle zone postapocalittiche delle fabbriche abbandonate. Il Pittore riprende la modalità della scomposizione e ricomposizione dei dettagli delle fotografie delle vittime fatte dal fotografo matto soprannominato Nikon, e rimanda a un utopistico progetto di ricomposizione della città dopo la scomposizione dei quartieri realizzata col colpo di Stato del Profeta. È una concezione artistica di ascendenza postmoderna su cui torneremo. E finisce per diventare un procedimento di creazione che viene applicato in tutti i campi, l'estetica, la politica, la genetica. A me fa pensare al già nominato Frankenstein di Mary Shelley ma soprattutto ai manifesti dei film con Boris Karloff, con quel mostro pieno di cicatrici che sembrava proprio un puzzle di organi presi da corpi diversi. Anche qui, la fuga citazionistica è infinita: in un altro momento del libro appare un quadro che somiglia a quelli di Fontana, con quella sua poetica del taglio che crea. E anche qui, come dici tu, appare il gioco fra riferimento highbrow e lowbrow, l'horror e l'arte contemporanea, il romanzo e i cartelloni dei film: il romanzo è terrificante, ma si nutre di pezzi che sono a volte colti a volte popular, a volte serissimi a volte divertenti. E il cerchio si chiude: la poetica del Pittore è la poetica di Beppe Devalle, ma è alla fine anche la poetica che guida la composizione del romanzo che stai leggendo...

\section{Monica JANSEN}

Mi ha colpito che la continuità tra le narrazioni di Eva e Avrai $i$ miei occhi si costruisca anche attraverso il gioco delle citazioni. Un bell'esempio è il riferimento esplicito a una canzone di De André fatto in Eva che diventa il ricordo di una canzone già non più presente nel futuro distopico progredito in Avrai 
i miei occhit $i^{14}$. Un altro esempio del gioco dei rimandi nascosti è una frase in Avrai $i$ miei occhi in cui si descrive la zona della "Città fuori delle mura": "E l'alba è un territorio comanche, dove non comanda nessuno e tutti vivono una festosa quanto breve anarchia" 45 . Territorio comanche è anche il titolo di un romanzo sulla guerra in Bosnia di Arturo Pérez-Reverte, pubblicato nel 1994, da cui fu tratto un film nel 1997. Così si stabilisce di nuovo un nesso con il romanzo precedente Eva che aveva come contesto storico il trauma delle guerre nella ex Iugoslavia. Un uso più programmatico dell'intertestualità è invece quello della riscrittura di certi archetipi patriarcali in chiave femminista. Oltre a quello biblico di Eva, ne è un altro esempio la ricorrenza quasi ossessiva della fiaba di Barbablù di Charles Perrault, che attraverso la mediazione dell'antologia The Bloody Chamber di Angela Carter scandisce il già citato racconto "Camere di sangue" in Mappe sulla pelle. Diviso in sei camere di sangue, è nella terza che il corpo della donna esce dalla stanza della tortura per conquistare la propria voce di scrittrice e appropriarsi della "stanza tutta per sé di Virginia Woolf"

Ho imparato a uscire da quella stanza, rubando la chiave a Barbablù per pugnalarlo col suo stesso coltello. [...] siamo noi le tessitrici. Siamo noi le narratrici di storie. Ho fatto quello che potevo per liberarle. Perché ho pensato che quello fosse il mio compito? Semplice e cristallino: perché io sono Angela Olive Stalker, la bambina sfollata, la ragazza dello Yorkshire, l'adolescente anoressica, la sposa giovane, la divorziata, la viaggiatrice, la femminista, la strega, la donna, la madre. Perché io sono Angela Carter, la narratrice di storie ${ }^{47}$.

La fiaba di Barbablù ricompare nelle storie di Eva e di Avrai $i$ miei occhi, nella prima per indicare la stanza dove i corpi uccisi da Eva vengono tagliati a pezzetti, e nella seconda invece per indicare la stanza segreta nel Palazzo dei Leoni dove viene custodito il corpo di Anastasia Darmus, la "cavia regina", e dove si

44. Olivia canta mentre trasporta Nigredo nel suo taxi: “'Se ti tagliassero a pezzetti il vento li raccoglierebbe...' Frenò e smise di cantare. E io aprii gli occhi. - De André. - Non te l'ho chiesto. - È una bella canzone. Lui è morto giovane" (VALlorani Nicoletta, Eva, cit., p. 130). In Avrai in miei occhi la stessa musica per Olivia ha perso i suoi connotati: "- Perché non ci hanno semplicemente tagliati a metà? - dico, col ricordo consapevole di una canzone nella voce. - Perché hanno dovuto farci a pezzetti?" (VALLORANI Nicoletta, Avrai i miei occhi, cit., p. 28).

45. Vallorani Nicoletta, Avrai i miei occhi, cit., p. 67.

46. Tessere Trame, "Una voce tutta per sé", cit.

47. Vallorani Nicoletta, "Camere di sangue", cit. Si noti un'altra assonanza intertestuale tra il nome della personaggia Olivia e Olive, il vero nome di Angela Carter. 
compierà l'atto di liberazione per mano di Ariel e Olivia ${ }^{48}$. Qui ritengo interessanti le riflessioni sul realismo magico di Carter fatte da Anna Pasolini a proposito di Nights at the Circus (1984). Considerati dalla critica body texts, le narrazioni di Carter diventano uno spazio dove la corporeità acquista una dimensione paradossale che fonde ciò che ideologicamente viene scritto sul corpo con ciò che parte dalla materialità del corpo per trasformarsi in riscrittura sovversiva. Secondo Pasolini, i nuovi modelli di liberazione e agency proposti da Carter partono proprio da una "ri-significazione del corpo", con l'aiuto di "strumenti alternativi a quelli offerti da pratiche discorsive oppressive" e in questo modo il femminile potrà essere risollevato da "una posizione di subalternità e asservimento" "49. La duplicità tra iscrizione e ri-significazione è resa tangibile in Avrai i miei occhi dai tatuaggi, le rune del possesso iscritte nelle braccia delle cavie che vengono portate alla discarica. Una volta decifrate, esse permetteranno di ricomporre i corpi in un "collage finalmente dotato di senso" 50 .

4. L'intertestualità va compresa nel senso ampio di intermedialità e transmedialità. I personaggi di Vallorani mettono in scena le diverse arti, con un ruolo particolare per pittura, scultura e fotografia. Sono frequenti le riflessioni dei personaggi sul ruolo dell'opera d'arte come forma del delitto e come chiave per risolvere il mistero. L'omaggio a Beppe Devalle in Avrai i miei occhi mette al centro una conceżione "asincrona" della realtà, parola su cui Vallorani riflette insieme alla scrittrice Elisa Emiliani sul blog di Zona $42^{51}$. Come si collega questa dimensione transmediale, che comprende anche il richiamo cinematografico a scene e situazioni, alla vocaz̧ione alla libertà che la scrittrice rivendica in relazione alle sue scelte narrative, estetiche e politiche?

\section{Claudio Milanesi}

Questa concezione è messa in bocca al personaggio del Pittore, da cui la coerenza dell'opera. In questo senso, questo procedimento narrativo richiama

48. "La stanza di Barbablù, dove tutto è cominciato. Ariel è la moglie disobbediente, e tu sei il suo complice. Ariel è colei che userà la chiave proibita per entrare, e che vendicherà le sue sorelle" (VAllorani Nicoletta, Avrai i miei occhi, cit., p. 260).

49. Pasolini Anna, Vallorani Nicoletta, Corpi magici, cit., p. 32.

50. Vallorani Nicoletta, Avrai i miei occhi, cit., p. 265. Il codice identificato con il numero $28754901 / \mathrm{X}$ riporta a un "genocidio delle cose che, in mancanza di nomi migliori, rievoca la vergogna di un altro olocausto, ricordandoci che non facciamo che ripetere la nostra storia" (Ibid., p. 51).

51. "[T]utto, in questa storia, parte dai dipinti di Beppe Devalle, che sono figure asincrone e distorte per definizione e per scelta. Visi che si smontano, corpi che confliggono, e nel farlo raccontano storie dolorose ma istruttive", BERnARdoni Angela, "Le autrici di Zona 42", cit. 
quello di un altro romanzo in cui la narrazione si intreccia con la declinazione della poetica che l'ha prodotta, La carte et le territoire di Michel Houellebecq (2010), che è tutto incentrato, fra l'altro, sulla dinamica fra la mappa e il territorio che descrive, tema che ritorna anche in Avrai i miei occhi, dove la conservazione della memoria è affidata appunto a una mappa della Milano di prima dello "Split", la suddivisione della città in quartieri stagni.

Ma quello che mi preme sottolineare su questo punto è la coerenza tematica ed estetica di Avrai i miei occhi: il principio di ricomposizione delle identità dei corpi delle vittime è il filo che permette lo sviluppo dell'inchiesta sui film di tortura e sui corpi dei cloni. Nikon fotografa dettagli dei cadaveri ed è la loro composizione a rendere possibile la creazione di una sorta di corpo collettivo. La divisione della città in distretti separati è il programma della dittatura del Profeta, ed è contro questa misura che lottano i pochi resistenti rimasti. Fra scomposizione dei corpi e scomposizione dei quartieri si instaura una corrispondenza produttrice di senso. Come ho detto, il romanzo stesso avanza per blocchi narrativi separati. Il suo principio estetico è enunciato dal Pittore: "Segmento e ricompongo". Il racconto, la storia e l'inchiesta procedono nello stesso modo, dalla raccolta di pezzi separati fino a una visione generale. Questo processo di ricomposizione delle parti separate fonda la coerenza tematica e poetica del romanzo, dove il lettore stesso, condotto per tutto il testo tra frammenti di storie e rimandi che lo lasciano sempre sull'orlo dell'incomprensione, arriva alla fine a quella visione globale che alcuni segnali narrativi gli hanno annunciato in modo trasversale, quali per esempio l'ascesa alla cima della torre più alta che permette finalmente la vista globale della città. L'esito del romanzo è questo: nonostante l'immersione nella semiosfera espansa del citazionismo, alla fine tutto si tiene e abbiamo l'impressione di trovarci di fronte alla realizzazione di un programma tematico, estetico e narrativo del tutto coerente.

Monica JANSEN

Vallorani ha molta affinità con artisti contemporanei, come dimostrano i riferimenti alle opere di Anish Kapoor descritte in Eva - le installazioni 1000 Names e Wound - oltre ai quadri di Devalle in Avrai i miei occhi, e la scrittrice non nasconde neanche le sue antipatie ${ }^{52}$. Ma forse ancora più affine al suo

52. "Damien Hirst - un cialtrone" (Vallorani Nicoletta, Avrai i miei occhi, cit., p. 83). Nei "Ringraziamenti", si precisa: "Soprattutto, c'è Cross Point, la tela che affianca Sylvia Plath, Francesca Woodman e Virginia Woolf in un dipinto di grandissima potenza. Marilyn Monroe e Sarah Kane appaiono in altre circostanze. Le ho amate tutte perdutamente, queste donne, nei ritratti di Beppe” (Ibid., p. 267). 
immaginario in bianco e nero è l'arte della fotografia, che sta anche alla base del modo di lavorare di Devalle, descritto sopra da Claudio. Del quadro Cross Point viene narrato l'attimo in cui il Pittore (Devalle) dipinge "il viso di Francesca Woodman, in parte nascosto da una mano sollevata a coprire la bocca. La nudità indifesa di una delle artiste più importanti del secolo scorso, morta suicida a pochi anni eppure già lucente del suo talento, evoca senza rivelare" ${ }^{53}$. L'americana Woodman, che trascorse un periodo a Roma e i cui autoritratti degli inizi degli anni Ottanta sono stati interpretati soprattutto in chiave femminista, lavorava molto con gli specchi, creando un gioco di riflesso e di sparizione ${ }^{54}$ che è anche frequente nella narrazione di Avrai i miei occhi. Ogni volta che Olivia e Nigredo si guardano nello specchietto retrovisore del taxi di Olivia scoprono di essere cyborg composti da materiali eterogenei.

L'atto di scattare fotografie non è poi un gesto innocente ma un'arma a doppio taglio, visto che l'occhio della telecamera espone la vulnerabilità del soggetto ripreso e allo stesso tempo produce le prove per poter giungere a una riparazione del torto. Il fotografo Nikon si chiama così dopo essersi riscattato dalle torture a cui è stato sottoposto, ma Nigredo continua a chiamarlo con il suo vero nome, Jacob Montelli. Ciò stabilisce una parentela (ebrea?) con il fotografo il Gobbo nel racconto "L'ultimo scatto" che in verità si chiama Saul Marchand, e ha lasciato alle spalle un'infanzia fatta di proibizioni e punizioni ${ }^{55}$. Ambedue i personaggi usano la loro arte come un atto pietoso verso le vittime fotografate, anche se uno dalla parte della giustizia e l'altro commettendo un crimine, e nel loro tentativo di ricomporre vita e morte sullo stesso piano rievocano la spiegazione che Eva dà a Nigredo dell'opera 1000 Names di Kapoor: "L'idea è conciliare gli opposti. Aggirare la frattura che li separa componendoli nello stesso spazio" "56. Solo che alla morte non si sopravvive, come commenta giustamente Nigredo.

5. Parlare dell'opera d'arte in termini di unicità, replica e copia, e in termini di decostruzione e ricomposizione di pezzi, frammenti, mosaici, porta inevitabilmente a parlare del postmoderno, altro concetto nominato nella prefazione alla nuova edizione di Eva per

53. Ibid., p. 141.

54. BAdger Gerry, "Un posto nel mondo. Leggere Francesca Woodman", in PediCINI Isabella, Francesca Woodman. Gli anni romani tra pelle e pellicola, Roma, Contrasto, 2012, p. 8.

55. Vallorani Nicoletta, "L'ultimo scatto", in Si muore bambini, cit., p. 35.

56. EAD., Eva, cit., p. 193. 
descrivere i personaggi. Quali interpretazioni del postmoderno possiamo trovare nei romanzi di Vallorani?

\section{Claudio Milanesi}

Quando, in Avrai i miei occhi, fa dire al Pittore: "Nulla è nuovo davvero ma la possibilità di combinazione dei pezzi sono infinite" ${ }^{27}$, Vallorani annuncia apertamente la propria poetica postmoderna. L'idea che tutte le forme fossero state ormai create e l'unica forma di creazione fosse ormai la loro combinazione era alla base della lettura postmoderna dell'architettura; basti pensare alla Piaz?a d'Italia di Charles Moore a New Orleans (1978), dove convivono elementi di epoca romana e stilemi dell'età moderna. Questo principio compositivo venne poi adottato anche dal romanzo, e in questo l'esempio più chiaro resta Il nome della rosa (1980) di Umberto Eco, che era appunto combinazione di romanzo poliziesco, storico, filosofico e politico.

Ma contrariamente al postmoderno ironico che reagiva col distacco all'età dell'impegno, quello di Vallorani è sì citazionista, ma ritrova una solida e dichiarata vocazione etica e critica. Citare, destrutturare e ricomporre non è solo un gioco raffinato, non è solo un divertissement, leggiadro romanzo di romanzi. Pochi giorni fa ho seguito Raccontare la città, un convegno ideato da Vallorani ${ }^{58}$, in cui Giulia De Spuches, una ricercatrice palermitana, discuteva di una produzione RAI, Poveri noi (1998) di Gianni Amelio, definendola un "documentario di documentari". Il documentario, in bianco e nero, componeva una ricerca antropologica e storica sulle popolazioni meridionali all'epoca delle migrazioni attraverso spezzoni di interviste coeve, e anche in quel caso, per quanto ci trovassimo di fronte a un collage di citazioni, non era certo l'aspetto ludico a prevalere, ma quello conoscitivo e di critica sociale. In questo senso, mi sembra che il citazionismo di Vallorani sia solo formalmente postmoderno, ma che poi l'intenzione trascenda il puro gioco raffinato per ritrovare, attraverso, nel suo caso, lo schermo del genere, la funzione etica della scrittura. Per spiegarmi meglio direi che il citazionismo di Vallorani è classicamente postmoderno, ma la sensazione che provoca è che, piuttosto di distribuire riferimenti in un gioco autocompiaciuto atto ad arricchire formalmente solo il proprio intertesto, quest'ultimo romanzo di Vallorani sia l'opera di una scrittrice che "riceve continuamente

57. Ead., Avrai i miei occhi, cit., p. 134.

58. Docucity. Raccontare il reale, Milano, 13-14 maggio 2021. https://docucity.unimi. it/2021/04/raccontare-il-reale-narrazione-ricerca-e-didattica-con-il-cinema-documentario/. I video del convegno sono su You'Tube: https://www.youtube.com/ playlist?list=PLQSNM0L_rLWt5eDaBIrEuI9qkU0vjhxTH. 
messaggi dalla semiosfera", per usare un'espressione di Alessandro Carrera ${ }^{59}$, e crea la narrazione con questo alfabeto di riferimenti. Una riprova indiretta di tale lettura mi sembra stia proprio in una dichiarazione di Vallorani, che in una sua intervista ${ }^{60}$ racconta come ritenesse di aver immaginato la caratteristica della "sensitiva" Olivia e delle Regine di Avrai i miei occhi, cioè quella di sentire emozioni e dolori degli altri; e di aver scoperto, solo dopo aver scritto il romanzo, che un personaggio dalle stesse caratteristiche esisteva già in La parabola del seminatore (1993) della scrittrice di fantascienza femminista Octavia E. Butler, pubblicato quasi trent'anni prima di Avrai i miei occhi.

\section{Monica JANSEN}

In un'intervista con il blog Leggere distopico, alla domanda se non ha mai pensato a "un ribaltamento dei ruoli ovvero far sì che le vittime siano gli uomini", Vallorani risponde: "La mia idea di scrittura corrisponde a quel che scriveva Lyotard quando distingueva tra la Storia ufficiale, che di uomini racconta in abbondanza, e le piccole storie di chi non ha la 'stanza tutta per sé' della quale parlava Virginia Woolf. Per quanto lo si dimentichi troppo spesso, chi racconta ha un compito etico"61. Anche il postmoderno nella concezione di Vallorani è da intendere quindi in chiave femminista. Ciò vale anche per il riuso di uno dei concetti chiave del postmoderno critico, la "Historiographic Metafiction" di Linda Hutcheon, che da Pasolini in Corpi magici viene proposto come strategia narrativa per restituire la voce e il controllo della narrazione a personaggi considerati di norma marginali, aggiungendo alla strategia narrativa della parodia la materialità biologica del corpo femminile $e^{62}$. Fondamentale in questo rispetto è il cambio di prospettiva da Eva, in cui la vicenda è narrata dal punto di vista di Nigredo, a Avrai i miei occhi dove la voce narrante passa a Olivia.

6. Centrale nei due romanæi, e nell'immaginario di Vallorani, è la città di Milano, la polis che in Avrai i miei occhi si trasforma in una metropoli postapocalittica divisa con muri in zone numerate dentro la città che sono separate dalla "Cinta", dall'area dei "campi

59. CARrera Alessandro, "L'antico profeta che bussò alle porte del cielo", in Robinson, supplemento a la Repubblica, 15 maggio 2021. Carrera si riferisce a Bob Dylan.

60. Intervista a Colibri, registrata il 6 maggio 2020, https://www.facebook.com/ Colibrimilano/videos/647374699326738

61. Raimondi Elisa, "Mysterious Writer: Nicoletta Vallorani", Leggere distopico, 2 marzo 2020, http://www.leggeredistopico.com/2020/03/13/ mysterious-writer-nicoletta-vallorani/.

62. Pasolini Anna, Vallorani Nicoletta, Corpi magici, cit., p. 40. 
industriali" fuori della città, $i$ cui abitanti sono considerati "non cittadini'*3. Il territorio oltre i campi industriali esula da ogni tipo di amministrazione. Vallorani, anche da studiosa di letteratura inglese, si interessa molto dei rapporti tra topografia urbana e immaginazione letteraria: come vengono collegati questi elementi nelle distopie da lei immaginate?

\section{Claudio Milanesi}

"La città ha subito lo scempio dei confini"” ${ }^{64}$. Quando ho chiesto a Vallorani quali ragioni l'avessero spinta a scrivere un seguito di Eva, fra i tanti motivi, mi ha detto che era per lei un modo di portare ancora più avanti la trasformazione della città. Quello della città, e in particolare di Milano, è un altro di quei motivi che attraversano la sua opera. Nella serie dei noir, Milano era indagata nella sua esistenza attuale, con i suoi quartieri ben individuabili socialmente, con la libertà che ne caratterizza i cittadini, le contraddizioni e i contrasti che la segnano, il suo lato oscuro, quello in cui operava già la saldatura fra la città ufficiale della produzione e dell'imprenditoria e quella ctonia del crimine e della notte. Con gli anni, quello per la città è diventato un interesse non solo narrativo ma anche di studiosa, e non è un caso che la ritroviamo oggi a capo di un evento ricorrente come quello di Docucity, a cui ho fatto riferimento, un festival di creazione visuale sulle immagini della città la cui ultima edizione, dedicata a Raccontare il reale, era in realtà tutta incentrata sulle immagini dell'Altro che abita fra noi e che è anzi diventato una parte di noi: ragazzi neri cha parlano con accento lombardo, signore milanesi di origine etiopiche che vanno in viale Etiopia a discutere con gli abitanti per risemantizzare la toponomastica. Sembrava di essere in uno dei suoi romanzi.

Per tornare a Avrai i miei occbi, la Milano della sua fantascienza le serve per immaginare le conseguenze sulla trasformazione della città del logico sviluppo di certe tendenze urbanistiche già visibili in quella di oggi. Quella Milano dello Split, con i suoi quartieri isolati l'uno dall'altro e ognuno uniforme dal punto di vista della composizione sociale, è il vero incubo metropolitano delle città di oggi, che ne riproduce alla massima potenza certe sue tendenze già visibili: $\mathrm{i}$ quartieri ricchi chiusi su se stessi, quelli intermedi divisi per professione ma ormai definitivamente esclusi dal cerchio magico delle corporazioni difese dal potere politico, e poi le popolazioni nomadi e marginali, che sopravvivono appena in squat e capannoni occupati. È il domani? O è già così oggi? E quelle

63. Una "Mappatura provvisoria" contenuta nel "registro municipale" è inserita come parte dei capitoli non numerati che riportano materiali "documentali" nel racconto; Vallorani Nicoletta, Avrai i miei occhi, cit., pp. 131-32.

64. Ibid., p. 137. 
immagini post apocalittiche che sembrano uscite da La strada o da Mad Max, dove gli adepti della setta dei Martiri si danno fuoco, dove nella nebbia passa lento il carro coi cadaveri dei cloni, è il nostro domani, o è l'oggi della pandemia, o è addirittura il nostro passato, quello del carro dei monatti della peste dei Promessi sposi? La Milano di prima dello Split era sì, come dice Olivia, "Bellissima", ma cova da sempre dentro di sé il seme dell'apocalisse e della frammentazione.

\section{Monica JANSEN}

Per rispondere a questa domanda potrei fare di nuovo ricorso a quanto scritto da Vallorani sul corpo della città nella finzione di Carter, e riportare il riferimento a Burton Pike sul progressivo frammentarsi del concetto di comunità fino al divorzio tra i concetti di civitas e communitas, non più sentiti come complementari ma come opposti ${ }^{65}$. Questo processo di sgretolamento del tessuto sociale è sicuramente raffigurato nella Milano del 2030 in cui i personaggi marginali non sono ulteriormente specificati che come "un groviglio di panni", "zingari", "bambini", "Martiri", "schiavi" e "mourners" "Ogni tanto compare un personaggio multietnico con un nome, Curtiz per esempio, dai lineamenti afroitaliani, con cui si stabilisce un rapporto di amicizia o di solidarietà. Diversa era la situazione in Eva, dove Nigredo condivideva lo spazio abitativo in quel che rimaneva del carcere di San Vittore con Dessa, che morirà di peste, che raccontava storie e cucinava piatti esotici ${ }^{67}$. In Avrai $i$ miei occhi San Vittore rimane una zona franca perché paradossalmente conserva il ricordo della comunità:

San Vittore è terra contaminata. Chi viene a stare qui, prima o poi ci muore. [...] E tuttavia, a San Vittore la gente continua a venirci, come se guardare il cielo da dietro sbarre ancora intatte fosse un passatempo intrigante e un po' snob. [...] E tra le mura, cantano ancora le storie di chi è stato chiuso nelle celle, in anni ormai remoti. Eppure tu lo sai che le case conservano i ricordi, come le persone, anche se non sono capaci di raccontarle in parole ${ }^{68}$.

65. Vallorani Nicoletta, "The Body of the City", cit., p. 366.

66. EAD., Avrai i miei occhi, cit., pp. 14, 79, 96, 10, 16, 10.

67. "Tutti e due, io e lei, abbiamo bisogno del carcere per funzionare a dovere. A San Vittore riusciamo a tirar fuori il meglio. Ammesso che sia un gran che" (VALLORANI Nicoletta, Eva, cit., p. 195).

68. Vallorani Nicoletta, Avrai i miei occhi, cit., p. 48. 
7. Non si parla solo di corpi, di pelle e di cicatrici, ma l'altro protagonista in queste narrażioni distopiche è il dolore. In Avrai i miei occhi la materia umana si complica e si decompone ulteriormente con un ingegnoso sistema criminale di abuso e profitto che mette sullo stesso piano oggetti e vita attraverso la creazione di cloni, cavie e cavie regina. Cosa aggiunge questa dimensione speculativa dell'oggettificazione del corpo femminile al discorso storico e politico sulla violenza contro le donne?

\section{Claudio Milanesi}

Non so se aggiunge qualcosa al discorso sulla violenza, quello che ho recepito è che la forza evocativa del romanzo è data dall'iterazione di una questione che resta aperta, lasciando quindi il lettore sconcertato e inappagato. Per spiegarmi meglio: tutti i protagonisti del romanzo partono dall'assunto che i cloni siano "cose". E che in quanto "cose" non provino sensazioni, non sentano dolore ed emozioni. Ma questo dogma che in qualche modo giustifica che se ne possa fare scempio è messo in discussione attraverso il legame empatico che collega i cloni alle cavie che, loro sì, provano dolore e sensazioni. E soprattutto, è messo in dubbio da un semplice interrogativo che ritorna nei dialoghi: siamo così sicuri che le cose non sentano? Alla domanda non c'è risposta. Il dubbio è però instillato. Intendiamoci: non è che ci venga il dubbio che un sasso soffra quando lo spacchiamo in tanti pezzi. Il punto è un altro: è la narrativizzazione della reificazione a essere in gioco. Da dove viene l'impunità che prova chi porta violenza sul corpo della donna? Dal considerare il corpo della donna un puro oggetto, dalla sua reificazione. Ma se la cosa sente, prova dolore?

Monica Jansen

A questa domanda Vallorani stessa dà una risposta chiara:

Qui l'idea - che è centrale nel romanzo, almeno nelle mie intenzioni - era quella di mostrare come il corpo femminile violato smetta di essere, nelle intenzioni di chi lo viola, una cosa organica. Esso diventa una cosa, in quanto tale subumana, e in quanto tale idonea a ogni genere di tortura. Con uno slittamento concettuale appena percepibile, ho usato una scienza consueta (anche se non ancora così sviluppata) per conferire a queste cose un'anima, e per ragionare un po' su come sia sfuggente questa autorizzazione alla violenza contro le donne. Mi premeva molto parlarne ${ }^{69}$.

Il bisogno etico di parlarne Vallorani lo mette in pratica non solo nelle sue storie di finzione, ma anche in testi di non finzione, come in una serie di rubriche sul

69. Contarini Silvia, “'Avrai i miei occhi', di Nicoletta Vallorani”, cit. 
blog Le parole e le cose dedicate al caso di Silvia Romano, in cui afferma che "la vittima è un corpo fragile, culturalmente, socialmente e politicamente ritenuto ininfluente, dimenticato con facilità, celebrato a intermittenza e strumentalmente alla funzione riproduttiva, mai ricordato e più spesso rimosso"70. Toccante infine il breve racconto "Leyla", pubblicato sul blog Sdiario, che mette in risalto la tragicità del fatto che queste donne sono state colpite nei loro talenti, per cui sono vittime e carnefici di se stesse allo stesso tempo. Dice Leyla:

Ero brava, intelligente, anche molto bella. Mi hanno ammessa al Gioco il 28 dicembre. Ero un dono prezioso per il nuovo anno, un pacchetto di vita per una persona importante, non ho mai saputo quale con precisione. Gli uomini che si sono occupati di me, armati di ogni genere di attrezzo, erano sei. Il mio tempo è stato 300 ore. Quasi un record ${ }^{71}$.

8. Nonostante la storia di violenza che attraversa queste finzioni, le guerre balcaniche in Eva e il femminicidio in Avrai i miei occhi, messo in relazione con il genocidio degli ebrei, sembra che ci sia ancora spazio per un'alternativa che risiede nell'arte di "ricomporre", come ci insegna il Pittore in Avrai i miei occhi. Come potremmo formulare la "resistenza" al vuoto di memoria, alla frammentazione del tessuto sociale e alle derive del neoliberalismo estraibile dalle congetture fin₹ionali di Vallorani?

\section{Claudio Milanesi}

È fin troppo facile parlare di Avrai i miei occhi come di un romanzo anarchico di critica radicale al neoliberalismo. Ma questa definizione seppur parziale è comunque pienamente giustificata da quanto abbiamo detto finora. Il nuovo mondo del Profeta è fondato sull'interesse di una casta protetta che ha ridotto il resto della società ai margini e fonda il proprio potere e il proprio arricchimento esponenziale sulla reificazione dei corpi, ridotti a nient'altro che puri strumenti del sistema. La resistenza passa dal sabotaggio e dal botto finale, e non dico altro per non svelare la fine del romanzo. In sintesi, una rappresentazione dei mali del mondo neoliberale condensata e portata alle sue massime conseguenze. E una narrativizzazione delle forme di resistenza preconizzate dai circoli anarchici, omaggiati qui col riferimento al Circolo della Ghisolfa,

70. Vallorani Nicoletta, "Negli interstizi della storia. Per Silvia Romano", Le parole e le cose, 29 maggio 2019, http://www.leparoleelecose.it/?p=35750.

71. Vallorani Nicoletta, "Leyla", Sdiario, 9 marzo 2020, https://sdiario.com/ leyla-di-nicoletta-vallorani/. 
il circolo di Giuseppe Pinelli, caduto dalla finestra della questura di Milano il 14 dicembre 1969 per un "malore attivo", come sentenziò la giustizia italiana.

Una cosa merita però di essere aggiunta, ed è un punto che viene lasciato sullo sfondo nel romanzo, prevedendone forse un seguito: sono le caratteristiche del regime che si è instaurato con l'avvento della dittatura del "Profeta". Il Profeta, di cui il lettore non sa granché, è arrivato al potere, a quanto pare di capire, con un colpo di Stato, ricordato qui come lo Split, la divisione, la spaccatura, il frazionamento di Milano. Ma il Profeta non è un dittatore classico del Novecento. È più simile a un Presidente degli alternative facts. Certo, si avvale delle Milizie, che esercitano la forza e garantiscono l'ordine. Ma il suo sistema di potere prevede anche i Sacerdoti, cui è invece delegata l'influenza sulle coscienze. E l'aspetto più interessante e contorto, e interessante perché contorto, è che ha approfittato di uno scandalo legato agli abusi sui corpi femminili per giustificare il colpo di Stato, salvo poi organizzare a proprio favore lo stesso commercio di carne femminile, ufficialmente negato ma diventato concretamente parte cruciale del sistema economico su cui fonda il suo potere. Manipolazione, fake news, alternative facts, interesse, sfruttamento. Niente di nuovo, in fondo. Dirà il Pittore:

- È stato un atto artistico? - chiedi. - Anche quello del Profeta?

- Non c'è stata ricomposizione. Solo tagli e nessuna sutura. - Riflette un attimo, il tuo amico, guardando la figura che ha composto e completandola poi con pochi tratti. - L'arte è sorpresa, assoluta imprevedibilità. Che cosa c'è di imprevedibile in quello che ha fatto il Profeta?

- Niente, lo so ${ }^{72}$.

\section{Monica JANSEN}

Le recensioni al romanzo da parte della critica femminista sottolineano la somiglianza delle pratiche solidali e sovversive descritte nei romanzi discussi con quelle che partono dall'opera seminale di Adriana Cavarero, Tu che mi guardi, tu che mi racconti. Filosofia della narrażione (2001). Così si legge nella recensione di Gloria Baldoni: "La sorellanza, la ricostruzione di genealogie e la distruzione dell'esistente sono le tattiche di resistenza escogitate da Olivia e Nigredo: tutte e tre sono pratiche femministe" 73 . Come si è visto, anche le analisi di Pasolini e Vallorani offrono delle chiavi per individuare le aperture nelle distopie. A

72. Vallorani Nicoletta, Avrai i miei occhi, cit., p. 137.

73. BALDONi Gloria, "Avrai i miei occhi di Nicoletta Vallorani (Zona42)”, Ghinea, agosto 2020, disponibile su http:/ / www.zona42.it/wordpress/avrai-i-miei-occhi-recensioni-8/ 
proposito della scrittrice di fantascienza statunitense Jemisin, si afferma che un'apocalisse non è mai totale e prevede sempre qualche tipo di ricomposizione:

Se l'apocalisse non è un evento definitivo, allora persino l'estinzione riguarda qualcuno e non tutti. Grusin rileva che è utile riflettere su che cosa accadrà dopo l'estinzione [...], considerato che essa si riferisce sempre ad alcune specie e non ad altre. Dunque, la questione è di cercare di immaginare in quale forma e con quale organizzazione sociale sarà possibile, per gli umani, sopravvivere" ${ }^{\text {"7 }}$.

Lo stesso vale per l'ultima frase, in corsivo, di Avrai i miei occhi con cui si chiude il romanzo dopo l'esplosione: "La città è respiro, e noi con lei". L'auspicio espresso prima da Nigredo, "Noi non moriamo, ma belle. Noi siamo salvi, andiamo a casa" lascia supporre che siano i due protagonisti a respirare con la città, e noi lettori, con loro.

Restano ancora da analizzare le cartografie delle mappe, la memoria dell'archivio anarchico, il rovesciamento del punto di vista, la poetica delle rovine e la topografia dell'assenza, il pulviscolo... Lasciamo tutto questo, e tanto altro, per le Conversation pieces, part 2.

Monica JANSEN

Universiteit Utrecht

Claudio Milanesi Aix Marseille Université, CAER, Aix-en-Provence, France

74. Pasolini Anna, Vallorani Nicoletta, Corpi magici, cit., p. 168. Il riferimento è a Grusin Richard (a cura di), A Multidisciplinary Exploration of Extinction and What Comes Next, Minneapolis, University of Minnesota Press, 2018, p. 20.

75. Vallorani Nicoletta, Avrai i miei occhi, cit., p. 265. 
